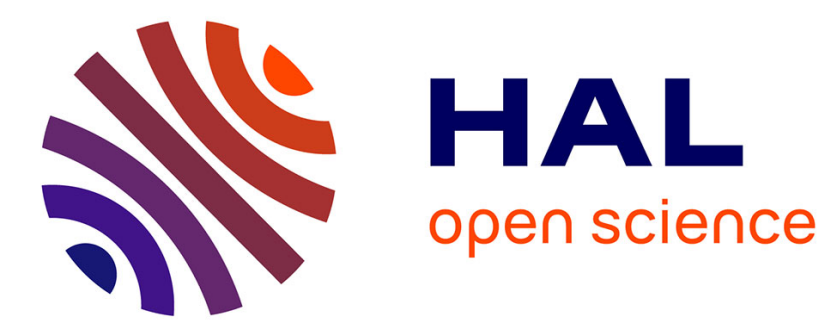

\title{
Multi-input nonlinear control systems linearizable via one-fold reduction
}

Florentina Nicolau, Shunjie Li, Witold Respondek

\section{To cite this version:}

Florentina Nicolau, Shunjie Li, Witold Respondek. Multi-input nonlinear control systems linearizable via one-fold reduction. The 37th Chinese control conference, Jul 2018, Wuhan, China. 10.23919/ChiCC.2018.8483716 . hal-01875437

\section{HAL Id: hal-01875437 https://hal.science/hal-01875437}

Submitted on 17 Sep 2018

HAL is a multi-disciplinary open access archive for the deposit and dissemination of scientific research documents, whether they are published or not. The documents may come from teaching and research institutions in France or abroad, or from public or private research centers.
L'archive ouverte pluridisciplinaire HAL, est destinée au dépôt et à la diffusion de documents scientifiques de niveau recherche, publiés ou non, émanant des établissements d'enseignement et de recherche français ou étrangers, des laboratoires publics ou privés. 


\title{
Multi-input nonlinear control systems linearizable via one-fold reduction
}

\author{
Florentina Nicolau ${ }^{1}$, Shunjie $\mathrm{Li}^{2}$, Witold Respondek ${ }^{3}$ \\ 1. QUARTZ Laboratory, ENSEA, 6 Avenue du Ponceau, 95014 Cergy-Pontoise, France. \\ E-mail: florentina.nicolau@ensea.fr \\ 2. School of Mathematics and Statistics, Nanjing University of Information Science and Technology, Nanjing, 201124, China. \\ E-mail: shunjie.li@ nuist.edu.cn \\ 3. Normandie Universié, INSA de Rouen, Laboratoire de Mathématiques, 76801 Saint-Etienne-du-Rouvray, France. \\ E-mail: witold.respondek@insa-rouen.fr
}

\begin{abstract}
In this paper we study the feedback linearization of multi-input control-affine systems via a particular class of nonregular feedback transformations. We give a complete geometric characterization of systems that become static feedback linearizable after a one-fold reduction of a suitably chosen control. That problem can be seen as the dual of the linearization via invertible one-fold prolongation of a suitably chosen control (which is the simplest dynamic feedback). We discuss in detail similarities and differences of both problems. We propose necessary and sufficient conditions describing the class of systems linearizable via a one-fold reduction, and discuss when the proposed conditions can be verified (by differentiation and algebraic operations only). We provide a normal form and illustrate our results by several examples.
\end{abstract}

Key Words: Feedback linearization, nonregular feedback, one-fold reduction, normal form.

\section{Introduction}

Feedback linearization is a powerful tool for nonlinear control systems and has attracted a lot of research in the recent years. Following the work of Brockett [1] who solved the state feedback linearization for single-input systems under a restricted class of feedback transformations, Jakubczyk and Respondek [11] and, independently, Hunt and Su [8] gave geometric necessary and sufficient conditions for linearizing multi-input affine control systems under change of coordinates and general static feedback transformations modifying both the drift and the control vector fields.

A more general class of feedback transformations is that of dynamic feedback transformations and the problem of linearization under such transformations was studied, for instance, in $[3,4,9,10,17]$. A closely related notion is that of flatness introduced in [5, 6], see also [12] and the references therein. It is well known that systems linearizable via invertible static feedback are flat. With the exception of the single-input case, where flatness reduces to static feedback linearization, see [3] and [17], a flat system is, in general, not linearizable by static feedback but may become so after applying a preliminary dynamic feedback (preintegration). So a flat system which is not static but dynamic feedback linearizable, can be seen as the reduction of a static feedback linearizable one. In this paper we consider a dual perspective of that observation. We study the following question: is a given nonlinear control system an extension (or a perturbation) of a static feedback linearizable one? More precisely, we deal with nonlinear control systems that steam from a system with less inputs which is, contrary to the original one, static feedback linearizable. This question is of practical interest since by identifying the inputs that make the systems non static feedback linearizable and removing them, one can plan and track trajectories for the reduced static feedback linearizable system. The goal of this paper

Research partially supported by the Natural Science Foundation of China (61573192). is thus to give a geometric characterization of control-affine systems that become static feedback linearizable after a onefold reduction of a suitably chosen control (we say that those systems are linearizable via one-fold reduction). That problem can be seen as the dual of the linearization via invertible one-fold prolongation: the simplest flat systems that are not static feedback linearizable are those that become linearizable via invertible one-fold prolongation of a suitably chosen control (which is the simplest dynamic feedback). That class of systems was completely characterized in [15] (see also [14]). The conditions for linearization via one-fold reduction reminds very much those for linearization via invertible one-fold prolongation. We discuss in detail similarities and differences of both problems.

Another closely related notion is that of nonregular feedback linearization (where the considered feedback transformations are not invertible), see, for example, [7, 19, 20]. Indeed, linearizarion via one-fold reduction can be seen as feedback linearization via a nonregular feedback which is "minimally noninvertible" (that is, the rank of the matrix defining the feedback transformation equals $m-1$, where $m$ is the number of controls, and is the maximal possible among all noninvertible matrices). Thus we deal with a problem of feedback linearization via a nonregular feedback but which remains as close as possible to a regular one.

The paper is organized as follows. In Section 2, we formalize the problem. In Section 3, we give our main results: we characterize control-affine systems linearizable via a one-fold reduction of a suitably chosen control. We provide necessary and sufficient conditions and explain how to verify them. We present a normal form describing the considered class of systems and discuss the construction of the control that has to be canceled in order to obtain a reduced static feedback linearizable system. We illustrate our results by several examples in Section 4. All proofs and additional comments are presented in the complete version [13]. 


\section{Problem statement}

Consider the following nonlinear control-affine system:

$$
\Sigma_{m}: \dot{x}=f(x)+\sum_{i=1}^{m} u_{i} g_{i}(x)=f(x)+g(x) u,
$$

where $x$ is the state defined on a open subset $X$ of $\mathbb{R}^{n}$ and $u$ is the control taking values in an open subset $U$ of $\mathbb{R}^{m}$ (more generally, an $n$-dimensional manifold $X$ and an $m$-dimensional manifold $U$, respectively). The vector fields $f, g_{1}, \ldots, g_{m}$ are smooth and the word smooth will always mean $\mathcal{C}^{\infty}$-smooth. The system $\Sigma_{m}$ is linearizable by static feedback if it is equivalent, via a diffeomorphism $z=\phi(x)$ and an invertible static feedback transformation $u=\alpha(x)+\beta(x) v$, to a linear controllable system $\Lambda: \dot{z}=A z+B v$. The problem of static feedback linearization was solved by Brockett [1] (for a smaller class of transformations) and then by Jakubczyk and Respondek [11] and, independently, by Hunt and Su [8], who gave geometric necessary and sufficient conditions (recalled in Theorem 1). Define inductively the sequence of distributions $\mathcal{D}^{i+1}=\mathcal{D}^{i}+\left[f, \mathcal{D}^{i}\right]$, where $\mathcal{D}^{0}=\operatorname{span}\left\{g_{1}, \ldots, g_{m}\right\}$ and denote $\left[f, \mathcal{D}^{i}\right]=\left\{[f, \xi]: \xi \in \mathcal{D}^{i}\right\}$.

\section{Theorem 1 ([8, 11]). The following are equivalent:}

(FL1) $\Sigma_{m}$ is locally static feedback linearizable, around $x_{0} \in X$

(FL2) $\Sigma_{m}$ is locally static feedback equivalent, around $x_{0} \in$ $X$, to the Brunovský canonical form

$$
(B r):\left\{\begin{array}{l}
\dot{z}_{i}^{j}=z_{i}^{j+1} \\
\dot{z}_{i}^{\rho_{i}}=v_{i}
\end{array}\right.
$$

where $1 \leq i \leq m, 1 \leq j \leq \rho_{i}-1$, and $\sum_{i=1}^{m} \rho_{i}=n$; (FL3) For any $0 \leq q \leq n-1$, the distributions $\mathcal{D}^{q}$ are of constant rank, around $x_{0} \in X$, involutive, and $\mathcal{D}^{n-1}=T X$;

The geometry of static feedback linearizable systems is given by the following sequence of nested involutive distributions:

$$
\mathcal{D}^{0} \subset \mathcal{D}^{1} \subset \cdots \subset \mathcal{D}^{n-1}=T X .
$$

Static feedback linearization is a powerful tool in dealing with nonlinear systems and has been applied to many engineering systems, in particular, to the problems of constructive controllability and motion planning. Although, in general, a nonlinear control system is not static feedback linearizable, it may steam, however, from a system with less inputs which is static feedback linearizable.

More precisely, the problem that we are addressing in this paper is the existence of a local invertible static feedback transformation of the form

$$
u=\beta^{r e g}(x) \tilde{u}, \quad \operatorname{rank} \beta^{r e g}(\cdot)=m,
$$

bringing the system $\Sigma_{m}$ into

$$
\tilde{\Sigma}_{m}: \dot{x}=f(x)+\sum_{i=1}^{m} \tilde{u}_{i} \tilde{g}_{i}(x),
$$

with $\tilde{g}=g \beta^{r e g}$, where $g=\left(g_{1}, \ldots, g_{m}\right)$ and $\tilde{g}=$ $\left(\tilde{g}_{1}, \ldots, \tilde{g}_{m}\right)$ are such that the reduced system

$$
\widetilde{\Sigma}_{m-1}: f(x)+\sum_{i=1}^{m-1} \tilde{u}_{i} \tilde{g}_{i}(x)
$$

is locally invertible static feedback linearizable. A system $\Sigma_{m}$ satisfying the above property will be called linearizable via one-fold reduction. Indeed, the system $\widetilde{\Sigma}_{m-1}$ is, as indicated by the notation, obtained by removing the control $\tilde{u}_{m}$ (for which we put $\tilde{u}_{m} \equiv 0$ ) and keeping $\tilde{u}_{i}$, for $1 \leq i \leq m-1$, unchanged. The feedback transformation $u=\beta(x) \tilde{u}$ that defines the passage from $\tilde{\Sigma}_{m}$ into $\widetilde{\Sigma}_{m-1}$ is given by $\beta$ consisting of first $m-1$ columns of $\beta^{\text {reg }}$.

Notice that when linearizing $\widetilde{\Sigma}_{m-1}$ with the help of $\tilde{u}=$ $\tilde{\alpha}(x)+\tilde{\beta}(x) v$, it is actually enough to apply the pure feedback $\tilde{u}=\tilde{\alpha}(x)+v$ only (that simply transforms $f$ into $f+\sum_{i=1}^{m-1} \tilde{\alpha}_{i} \tilde{g}_{i}$ ) because changing $\tilde{g}_{i}$ 's, for $1 \leq i \leq m-1$, can be performed via the initial nonregular feedback $\beta$ (the one that consists of first $m-1$ columns of $\beta^{\text {reg }}$ and allows to eliminate the control $\tilde{u}_{m}$ from the system). In other words, instead of applying a nonregular $\beta$ (of rank $m-1$ ) followed by an invertible $\tilde{\beta}$ of rank $m-1$, we can just apply one nonregular $\beta$ (of rank $m-1$ ) that plays a double role.

To summarize, our problem of linearization via one-fold reduction can be equivalently formulated as follows: when the nonlinear control system $\dot{x}=f+g u$, is equivalent via a diffeomorphism $z=\phi(x)$ and a feedback transformation of the form $u=\beta(v+\tilde{\alpha})$, where $\operatorname{rank} \beta(\cdot)=m-1$, to a linear controllable system $\Lambda: \dot{z}=A z+B v$.

The subject of our paper is closely related to the slightly more general problem of linearization via noninvertible feedback transformations, see [7, 19, 20]. To compare both problems, notice that the class of feedback transformations (for linearization via one-fold reduction) considered in this paper is not as general as possible. Indeed, we use feedback transformations of the form

$$
u=\beta(v+\tilde{\alpha}),
$$

where $\operatorname{rank} \beta(\cdot)=m-1$ and $\tilde{\alpha}$ is an $\mathbb{R}^{m-1}$-valued function, that is, we apply to the original system $\Sigma_{m}$ first $\beta$ and then $\tilde{\alpha}$ and get $\dot{x}=f+g \beta \tilde{\alpha}+g \beta v$. If the matrix $\beta(\cdot)$ were invertible, the order in which we apply $\alpha$ and $\beta$ would play no role but if the matrix $\beta(\cdot)$ is not invertible, then the order does matter. Indeed, if we apply first $\alpha$ and then $\beta$, that is, we put

$$
u=\alpha+\beta v,
$$

where $\operatorname{rank} \beta(\cdot)=m-1$ but $\alpha$ is an $\mathbb{R}^{m}$-valued function, then the modified system is $\dot{x}=f+g \alpha+g \beta v$. For both classes of transformations, we choose $m-1$ new control vector fields $\tilde{g}_{i}$, for $1 \leq i \leq m-1$, in the same way as $\tilde{g}_{i}=\sum_{j=1}^{m} \beta_{i}^{j} g_{j}$ but, clearly, the second class (which defines all noninvertible feedback transformations) is more general because it allows to modify the drift $f$ by any smooth combination of $g_{i}$ for $1 \leq i \leq m$, while the second allows to modify $f$ by adding to it smooth combinations of $\tilde{g}_{i}$ for $1 \leq i \leq m-1$ only. The first class is, however, more natural in all cases when we have to decrease the number of controls from $m$ to $m-1$ and, as a consequence, we are not allowed to use in control strategies (in feedback transformations, for instance) all inputs but only those of the reduced system. We use it in the present paper.

Example 4 illustrates that, indeed, the two operations do not commute: it is more general to first apply $\alpha$ and then a noninvertible $\beta$ (consisting of an invertible $\beta^{\text {reg }}$ followed by a one-fold reduction) than to apply first the noninvertible $\beta$ and only then a function $\tilde{\alpha}$. 
Throughout, we make the following assumptions:

(A1) We assume that all ranks involved are constant in a neighborhood of a given $x_{0} \in X$. All results are valid on an open and dense subset of $X$ and hold locally, around any given point of that set.

(A2) We assume that the considered systems are accessible.

From now on, we deal only with systems that are not static feedback linearizable. Therefore one of the distributions $\mathcal{D}^{q}$ fails to satisfy condition (FL3) of Theorem 1. Indeed, the system is assumed accessible so $\mathcal{D}^{n-1}=T X$ holds and all distributions $\mathcal{D}^{q}$ are supposed to be of constant rank, see assumptions (A1)-(A2) above. So there exists an integer $q$ such that $\mathcal{D}^{q}$ is not involutive. Before giving our main results, let us introduce the notion of corank that will be used in the paper.

Notation 1. Let $\mathcal{A}$ and $\mathcal{B}$ be two distributions of constant rank and $f$ a vector field. Denote $[\mathcal{A}, \mathcal{B}]=\{[a, b]: a \in \mathcal{A}$, $b \in \mathcal{B}\}$ and $[f, \mathcal{B}]=\{[f, b]: b \in \mathcal{B}\}$. If $\mathcal{A} \subset \mathcal{B}$, the corank of the inclusion $\mathcal{A} \subset \mathcal{B}$, denoted by cork $(\mathcal{A} \subset \mathcal{B})$, equals the rank of the quotient $\mathcal{B} / \mathcal{A}$, i.e., $\operatorname{cork}(\mathcal{A} \subset \mathcal{B})=$ $\operatorname{rk}(\mathcal{B} / \mathcal{A})$.

\section{Main results}

Our main result is given by the following theorem that provides necessary and sufficient geometric conditions for linearization via one-fold reduction.

Theorem 2. $\Sigma_{m}$ is locally linearizable, around $x_{0}$, via a one-fold reduction if and only if it satisfies around $x_{0}$ :

(RI) There exists an involutive subdistribution $\mathcal{N}^{0} \subset \mathcal{D}^{0}$, of corank one;

(R2) The distributions $\mathcal{N}^{i}$, for $i \geq 1$, are involutive, where $\mathcal{N}^{i}=\mathcal{N}^{i-1}+\left[f, \mathcal{N}^{i-1}\right]$, for $i \geq 1$;

(R3) There exists $\rho$ such that $\mathcal{N}^{\rho}=T X$.

The conditions of the above theorem recall very much those for linearization via invertible one-fold prolongation, or, equivalently, for flatness of differential weight $n+m+1$ (see [18] for the definition of the differential weight of a flat system, and [15] for a complete geometric characterization of flat systems of differential weight $n+m+1$, where $n$ is the state dimension and $m$ is the number of controls). For those systems we have, as for the class described by Theorem 1, a sequence of inclusions of nested distributions. The most important structural condition characterizing systems linearizable via invertible one-fold prolongation is the existence of an involutive subdistribution $\mathcal{H}^{k}$ of corank one in $\mathcal{D}^{k}$ (which is the first noninvolutive distribution of the sequence $\mathcal{D}^{i}$ associated to the original $m$-input control system $\Sigma_{m}$ ). If $k \geq 1$, then, starting from $\mathcal{H}^{k}$, we can construct an increasing sequence of involutive distributions $\mathcal{H}^{i}=\mathcal{H}^{i-1}+\left[f, \mathcal{H}^{i-1}\right]$, for $i \geq k+1$, as well as a decreasing sequence of involutive distributions $\mathcal{H}^{i}=\mathcal{D}^{i-1}+\left[f, \mathcal{H}^{i-1}\right]$, for $i \geq 1$, with $\mathcal{H}^{0}$ being an involutive subdistribution of corank one in $\mathcal{D}^{0}$ uniquely associated to $\mathcal{H}^{k}$. It can be shown that, for $i \geq 2$, we actually have $\mathcal{H}^{i}=\mathcal{H}^{i-1}+\left[f, \mathcal{H}^{i-1}\right]$. If $k=0$, then, the first noninvolutive distribution is $\mathcal{D}^{0}$ and it has to contain an involutive subdistribution of corank one $\mathcal{H}^{0}$. Now, the sequence $\mathcal{H}^{i}$ is defined by $\mathcal{H}^{1}=\mathcal{D}^{0}+\left[\mathcal{D}^{0}, \mathcal{D}^{0}\right]+\left[f, \mathcal{H}^{0}\right]$ and
$\mathcal{H}^{i}=\mathcal{D}^{i-1}+\left[f, \mathcal{H}^{i-1}\right]$, for $i \geq 2$.

At first glance the distributions $\mathcal{N}^{i}$ and $\mathcal{H}^{i}$ seem identical, but, in general, only $\mathcal{N}^{0}$ and $\mathcal{H}^{0}$ may be the same and that is always the case if $\mathcal{D}^{0}$ contains a unique involutive subdistribution of corank one (that is, when $\mathcal{D}^{0}$ is noninvolutive and cork $\left(\mathcal{D}^{0} \subset \mathcal{D}^{0}+\left[\mathcal{D}^{0}, \mathcal{D}^{0}\right]\right) \geq 2$, see Section 3.2). Even if $\mathcal{N}^{0}$ and $\mathcal{H}^{0}$ are the same, the distributions $\mathcal{N}^{i}$ and $\mathcal{H}^{i}$, for $i \geq 1$, are, in general, different since the construction of $\overline{\mathcal{H}}^{1}$ as $\mathcal{H}^{1}=\mathcal{D}^{0}+\left[f, \mathcal{H}^{0}\right]$, if $k \geq 1$ (resp., as $\mathcal{H}^{1}=\mathcal{D}^{0}+\left[\mathcal{D}^{0}, \mathcal{D}^{0}\right]+\left[f, \mathcal{H}^{0}\right]$, if $k=0$ ) implies that the vector field completing $\mathcal{N}^{0}$ to $\mathcal{D}^{0}$ (and its successive brackets with the drift) is necessarily present in all the distributions $\mathcal{H}^{i}$ (contrary to $\mathcal{N}^{i}$ which are never directly affected by that vector field neither by its brackets with the drift). Indeed, suppose that $k \geq 1$, the distributions $\mathcal{H}^{0}$ and $\mathcal{N}^{0}$ coincide and the vector fields $g_{1}, \ldots, g_{m}$ are such that $\mathcal{H}^{0}=\mathcal{N}^{0}=\operatorname{span}\left\{g_{1}, \ldots, g_{m-1}\right\}$ and $\mathcal{D}^{0}=\operatorname{span}\left\{g_{1}, \ldots, g_{m}\right\}$. Using these notations, we have $\mathcal{N}^{i}=\operatorname{span}\left\{g_{j}, \ldots, a d_{f}^{i} g_{j}, 1 \leq j \leq m-1\right\}$ and $\mathcal{H}^{i}=\mathcal{N}^{i}+\operatorname{span}\left\{g_{m}, \ldots, a d_{f}^{i-1} g_{m}\right\}$, for $i \geq 1$. Of course, the new directions of the sequence $\mathcal{H}^{i}$ gained with the help of $g_{m}$ have to appear also in the sequence $\mathcal{N}^{i}$ from a certain rank $\ell$ (since $\mathcal{N}^{\rho}=T X$ ). Those directions are added by vector fields satisfying, for instance, a relation of the form $a d_{f}^{\ell} g_{q}=\alpha_{q} g_{m} \bmod \mathcal{N}^{\ell-1}+\operatorname{span}\left\{a d_{f}^{\ell} g_{j}, 1 \leq j \leq\right.$ $m-1, j \neq q\}$, where $1 \leq q \leq m-1$ and $\alpha_{q}\left(x_{0}\right) \neq 0$. If this happens starting from the smallest possible rank $\ell$ (i.e., from $\ell=1$ ), we actually have $\mathcal{H}^{1}=\mathcal{N}^{1}$ yielding $\mathcal{H}^{i}=\mathcal{N}^{i}$, for $i \geq 2$ (see Example 2). Therefore, in the case when $\mathcal{N}^{0}$ and $\mathcal{H}^{0}$ are the same, the relations between the sequences $\mathcal{N}^{i}, \mathcal{H}^{i}$ and $\mathcal{D}^{i}$ can be summarized by the following sequence of inclusions:

$$
\begin{aligned}
& \mathcal{D}^{0} \subset \mathcal{D}^{1} \cdots \subset \mathcal{D}^{k} \\
& 1 \cup \quad 1 \cup \quad 1 \cup \\
& \begin{array}{lllllllllll}
\mathcal{H}^{0} & \subset & \mathcal{H}^{1} & \cdots & \subset & \mathcal{H}^{k} & \subset & \mathcal{H}^{k+1} & \cdots \subset & \mathcal{H}^{\mu}=T X
\end{array}
\end{aligned}
$$

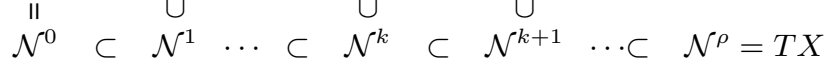

where all distributions, except $\mathcal{D}^{k}$, are involutive, cork $\left(\mathcal{H}^{i} \subset \mathcal{D}^{i}\right)=1$, for $0 \leq i \leq k$ (justifying the integer 1 appearing in front of the symbols " $\cup$ "), all inclusions $\mathcal{N}^{i} \subset \mathcal{H}^{i}$ are of corank at most $i$ and, in general, the integer $\rho$ is greater than $\mu$. The geometries of both classes of systems seem similar (even identical at first glance), but the two problems are structurally different. Obviously, there are systems that are at the same time linearizable via a one-fold reduction and linearizable via an invertible one-fold prolongation (see Examples 1-2), but in general, this is not the case (see, for instance, Example 3).

Similarly to systems linearizable via one-fold prolongation, for which we distinguished a to-be-prolonged control $u_{p}$, here we have to identify a to-be-removed control $u_{r}=\tilde{u}_{m}$ (whose removal leads to a locally static feedback linearizable reduced system). We explain in Section 3.1 the importance of $\mathcal{N}^{0}$ in computing $u_{r}$. The involutive subdistribution $\mathcal{N}^{0}$ plays a very important role for the class of system linearizable via one-fold reduction: with its help, we are able to construct the to-be-removed control and, moreover, successive brackets of the drift $f$ with $\mathcal{N}^{0}$ define the distributions $\mathcal{N}^{i}$ that are involutive and take the place of the (noninvolutive) distributions $\mathcal{D}^{i}$ associated to the original system. 


\subsection{To-be-removed control}

We construct in this section the control $u_{r}$ to be canceled by putting $u_{r} \equiv 0$ in order to obtain a static feedback linearizable reduced system. According to condition (R1) of Theorem 2, the distribution $\mathcal{D}^{0}$ contains a corank one subdistribution $\mathcal{N}^{0}$ that, as we will see, plays a crucial role in defining the to-be-removed control.

Since rk $\mathcal{N}^{0}=m-1$, we can find $m$ functions $\beta_{1}, \ldots, \beta_{m}$ (not vanishing simultaneously) such that $u_{1}(x) \beta_{1}(x)+\cdots+u_{m}(x) \beta_{m}(x)=0$ if and only if $\sum_{i=1}^{m} u_{i}(x) g_{i}(x) \in \mathcal{N}^{0}(x)$. The to-be-removed control $u_{r}$ (becoming $\tilde{u}_{m}$ after feedback) is given by

$$
u_{r}=\tilde{u}_{m}=u_{1} \beta_{1}(x)+\cdots+u_{m} \beta_{m}(x),
$$

Therefore $u_{r}$ is not unique and given up to multiplicative function. Indeed, if $u_{r}$ is a to-be-removed control, then so is $\hat{u}_{r}=u_{1} \hat{\beta}_{1}(x)+\cdots+u_{m} \hat{\beta}_{m}(x)$, where $\hat{\beta}_{i}=\gamma \beta_{i}$, with $\gamma(x) \neq 0$ arbitrary. What is thus canonical is not a to-be-removed control $u_{r}=\tilde{u}_{m}=u_{1} \beta_{1}(x)+\cdots+$ $u_{m} \beta_{m}(x)$ (resp. not the $\mathbb{R}^{m}$-valued vector function $\left(\beta_{1}(x)\right.$, $\left.\ldots, \beta_{m}(x)\right)$ defining it) but the collection of all to-beremoved controls $\gamma(x) u_{r}$, with $\gamma(x) \neq 0$, (resp. the field of lines $\left[\beta_{1}(x): \beta_{2}(x): \cdots: \beta_{m}(x)\right]$ in $\mathbb{R}^{m}$, where the latter denotes projective coordinates in $\mathbb{R}^{m}$ ). Finding $u_{r}$ requires knowing $\beta_{1}, \ldots, \beta_{m}$, which in turn is reduced to calculating $\mathcal{N}^{0}$. The latter problem is discussed in the next section. Notice that if $\mathcal{D}^{0}$ contains a unique involutive corank one subdistribution, then the to-be-removed control for linearization via one-fold reduction is the same (up to an affine transformation) as the to-be-prolonged control for linearization via invertible one-fold prolongation.

\subsection{Verification of the conditions}

In order to verify conditions (R1)-(R3) of Theorem 2, we have to check whether the distribution $\mathcal{D}^{0}$ contains an involutive subdistribution $\mathcal{N}^{0}$ of corank one. We will see that the corank $r$ of the inclusion $\mathcal{D}^{0} \subset \mathcal{D}^{0}+\left[\mathcal{D}^{0}, \mathcal{D}^{0}\right.$, equal to rk $\left(\left(\mathcal{D}^{0}+\left[\mathcal{D}^{0}, \mathcal{D}^{0}\right]\right) / \mathcal{D}^{0}\right)$, see Notation 1 , plays an important role in verifying our conditions. In fact, if $r \geq 2$, then the existence of $\mathcal{N}^{0}$ (and its construction, if it exists) is given by Proposition 1 below and we thus get verifiable necessary and sufficient conditions for linearization via one-fold reduction, stated as Theorem 3 below.

Consider a distribution $\mathcal{D}$ of rank $d$, defined on a manifold $X$ of dimension $n$ and define its annihilator $\mathcal{D}^{\perp}=$ $\left\{\omega \in \Lambda^{1}(X):\langle\omega, \xi\rangle=0, \forall \xi \in \mathcal{D}\right\}$, where $\Lambda^{1}(X)$ is the space of smooth differentials 1 -forms on $X$. Let $\operatorname{cork}(\mathcal{D} \subset \mathcal{D}+[\mathcal{D}, \mathcal{D}])=r$ and let $\omega_{1}, \ldots, \omega_{r}$, $\omega_{r+1}, \ldots, \omega_{s}$, where $s=n-d$, be differential 1-forms such that locally $\mathcal{D}^{\perp}=\operatorname{span}\left\{\omega_{1}, \ldots, \omega_{s}\right\}$ and $(\mathcal{D}+[\mathcal{D}, \mathcal{D}])^{\perp}=$ span $\left\{\omega_{r+1}, \ldots, \omega_{s}\right\}$. The Engel rank of $\mathcal{D}$ equals 1 at $x$ if and only if $\mathcal{D}$ is non involutive and $\left(d \omega_{i} \wedge d \omega_{j}\right)(x)=$ $0 \bmod \mathcal{D}^{\perp}$, for any $1 \leq i, j \leq s$. For any $\omega \in \mathcal{D}^{\perp}$, we define $\left.\mathcal{W}(\omega)=\{\xi \in \mathcal{D}: \xi\lrcorner d \omega \in \mathcal{D}^{\perp}\right\}$, where $\lrcorner$ is the interior product. The characteristic distribution $\mathcal{C}=\{\xi \in \mathcal{D}$ : $[\xi, \mathcal{D}] \subset \mathcal{D}\}$ of $\mathcal{D}$ is given by

$$
\mathcal{C}=\bigcap_{i=1}^{s} \mathcal{W}\left(\omega_{i}\right)
$$

It follows directly from the Jacobi identity that the characteristic distribution is always involutive. Define the distribution

$$
\mathcal{B}=\sum_{i=1}^{r} \mathcal{W}\left(\omega_{i}\right)
$$

Although the distributions $\mathcal{W}\left(\omega_{i}\right)$ depend on the choice of $\omega_{i}$ 's, the distribution $\mathcal{B}$ does not and we have the following result [16] based on [2].

Proposition 1. Consider a distribution $\mathcal{D}$ of rank $d$ and let $\operatorname{cork}(\mathcal{D} \subset \mathcal{D}+[\mathcal{D}, \mathcal{D}])=r$.

(i) Assume $r \geq 3$. The distribution $\mathcal{D}$ contains an involutive subdistribution $\mathcal{N}$ of corank one if and only if it satisfies

(ISD1) The Engel rank of D equals one;

(ISD2) The characteristic distribution $\mathcal{C}$ of $\mathcal{D}$ has rank $d-r-1$.

Moreover, that involutive subdistribution is unique and is given by $\mathcal{N}=\mathcal{B}$.

(ii) Assume $r=2$. The distribution $\mathcal{D}$ contains a corank one involutive subdistribution $\mathcal{N}$ if and only if $\mathcal{D}$ verifies (ISD1)-(ISD2) and the distribution $\mathcal{B}$ is involutive. Then $\mathcal{N}$ is unique and given by $\mathcal{N}=\mathcal{B}$.

(iii) Assume $r=1$. The distribution $\mathcal{D}$ contains an involutive subdistribution of corank one $\mathcal{N}$ if and only it satisfies condition (ISD2). In the case $r=1$, if an involutive subdistribution $\mathcal{N}$ of corank one exists, it is never unique.

(iv) Assume $r=0$. The distribution $\mathcal{D}$ always contains an involutive subdistribution of corank one $\mathcal{N}$ which is never unique.

The above conditions are easy to check and a unique involutive subdistribution of corank one can be constructed if $r \geq 2$, i.e., cork $(\mathcal{D} \subset \mathcal{D}+[\mathcal{D}, \mathcal{D}]) \geq 2$. Therefore, we can check (verifying (ISD1)-(ISD2) for $\mathcal{D}=\mathcal{D}^{0}$ and, only if $r \geq 2$, the involutivity of $\mathcal{B}$ ) whether an involutive subdistribution $\mathcal{N}^{0}$ of corank one in $\mathcal{D}^{0}$ exists and if so, then it is unique and can be explicitly calculated. As a consequence, for any given control-affine system satisfying cork $\left(\mathcal{D}^{0} \subset \mathcal{D}^{0}+\left[\mathcal{D}^{0}, \mathcal{D}^{0}\right]\right) \geq 2$, the conditions of Theorems 2 are verifiable and we can thus check whether the system is linearizable via one-fold reduction, as summarized by Theorem 3. Moreover, the verification involves differentiation and algebraic operations only, without solving PDE's or bringing the system into a normal form.

Theorem 3. Consider the control system $\Sigma_{m}$, given by (1), and suppose that cork $\left(\mathcal{D}^{0} \subset \mathcal{D}^{0}+\left[\mathcal{D}^{0}, \mathcal{D}^{0}\right]\right)=r \geq 2$. The system $\Sigma_{m}$ is linearizable via one-fold reduction, locally around $x_{0}$, if and only if $\mathcal{D}^{0}$ satisfies either item $(i)$, if $r \geq 3$, or item (ii), if $r=2$, of Proposition 1 and its unique involutive subdistribution $\mathcal{N}^{0}$, given by that proposition, fulfils conditions (R1)-(R3) of Theorem 2.

If $r \leq 1$, that is, cork $\left(\mathcal{D}^{0} \subset \mathcal{D}^{0}+\left[\mathcal{D}^{0}, \mathcal{D}^{0}\right]\right) \leq 1$, then, according to Proposition 1 (iii)-(iv), if an involutive subdistribution $\mathcal{N}^{0}$ of corank one of $\mathcal{D}^{0}$ exists, then it is never unique. It is easy to see that not all choices of an involutive subdistribution $\mathcal{N}^{0}$ lead to systems feedback linearizable via one-fold reduction. A natural question arises: how to identify the "right" $\mathcal{N}^{0}$ (that is, the subdistribution $\mathcal{N}^{0}$ that leads to a static feedback linearizable reduction) in the case $r=0$ and $r=1$ ?

For instance, if $r=1$, then an involutive subdistribution $\mathcal{N}^{0}$ of corank one in $\mathcal{D}^{0}$ exists if and only if the characteristic distribution $\mathcal{C}^{0}$ of $\mathcal{D}^{0}$ is of corank two in $\mathcal{D}^{0}$, see 
Proposition 1(iii). It is easy to check whether this last condition holds or not but the main difficulty is that if $\mathcal{N}^{0}$ exists, then it is never unique (see again Proposition 1(iii)) and we would have to verify for each involutive subdistribution $\mathcal{N}^{0}$ whether it satisfies conditions (R1)-(R3) of Theorem 2. There is no an algorithmic way to do it because the family of all involutive subdistributions $\mathcal{N}^{0}$ of corank one in $\mathcal{D}^{0}$ is parameterized by a functional parameter. Therefore, for the case cork $\left(\mathcal{D}^{0} \subset \mathcal{D}^{0}+\left[\mathcal{D}^{0}, \mathcal{D}^{0}\right]\right) \leq 1$, the involutive corank subdistribution $\mathcal{N}^{0}$ should be identified by another argument and this will be treated elsewhere.

\subsection{Feedback invariance}

For a static feedback linearizable (resp., linearizable via one-fold prolongation) system, the corresponding involutive distributions $\mathcal{D}^{i}$ (resp., $\mathcal{H}^{i}$ ) are feedback invariant. A natural question arises for systems linearizable via onefold reduction: under which feedback transformations are the distributions $\mathcal{N}^{i}$ invariant? Consider the control system $\Sigma_{m}$, given by (1). Apply an invertible feedback $u=$ $\beta(x) \tilde{u}$ such that $\mathcal{N}^{0}=\operatorname{span}\left\{\tilde{g}_{1}, \ldots, \tilde{g}_{m-1}\right\}$ and $\mathcal{D}^{0}=$ span $\left\{\tilde{g}_{1}, \ldots, \tilde{g}_{m-1}, \tilde{g}_{m}\right\}$, where $\tilde{g}=g \beta$. It is easy to see that the conditions of Theorem 2 are feedback invariant under feedback transformations of the form $\tilde{u}=\tilde{\beta}(x) \hat{u}$, where the invertible $(m \times m)$-matrix $\tilde{\beta}$ is such that $\tilde{\beta}_{m j}(x)=$ $0,1 \leq j \leq m-1$. Indeed, we clearly have $\mathcal{N}^{0}=$ $\operatorname{span}\left\{\tilde{g}_{1}, \ldots, \tilde{g}_{m-1}\right\}=\operatorname{span}\left\{\hat{g}_{1}, \ldots, \hat{g}_{m-1}\right\}$ and since the drift cannot change, the involutivity of $\mathcal{N}^{0}$ implies that $\mathcal{N}^{1}$ is feedback invariant. By an induction reasoning, it can be shown that all $\mathcal{N}^{i}$ are feedback invariant.

\subsection{Normal form}

The following proposition gives a normal form for system linearizable via one-fold reduction.

Proposition 2. The system $\Sigma_{m}$ is locally linearizable, around $x_{0}$, via a one-fold reduction if and only if it is locally, around $x_{0}$, equivalent via a diffeomorphism $z=\phi(x)$ and an invertible transformation $u=\beta^{r e g}(x) \tilde{u}$, where $\operatorname{rank} \beta^{r e g}(\cdot)=m$, to the following normal form in a neighborhood of $z_{0} \in \mathbb{R}^{n}$ :

$(N F)_{m}:\left\{\begin{array}{lll}\dot{z}_{i}^{j}=z_{i}^{j+1}+a_{i}^{j}(z) \tilde{u}_{m}, & 1 \leq j \leq \eta_{i}-1, \\ \dot{z}_{i}^{\eta_{i}}=f_{i}^{\eta_{i}}(z)+\tilde{u}_{i}, & & 1 \leq i \leq m-1,\end{array}\right.$

where $\Sigma_{i=1}^{m-1} \eta_{i}=n$, the functions $a_{i}^{j}$ are smooth, not all vanishing simultaneously at $z_{0}$, and such that at least one distribution $\mathcal{D}^{i}$ is not involutive.

The normal form $(N F)_{m}$ is clearly locally linearizable via one-fold reduction. Indeed, the reduced system $(N F)_{m-1}$ (obtained by canceling the control $\tilde{u}_{m}$ as $\tilde{u}_{m} \equiv$ 0 ) is locally static feedback linearizable by applying $v_{i}=$ $f_{i}^{\eta_{i}}(z)+\tilde{u}_{i}, 1 \leq i \leq m-1$. The presence of the nonlinear terms $f_{i}^{\eta_{i}}(z)$ is due to the fact that the drift of the original system $\Sigma_{m}$ is not modified if we apply feedback transformations of the form $u=\beta^{r e g}(x) \tilde{u}$. The normal form $(N F)_{m}$ is similar to the Brunovský canonical form $(B r)$, the differences being the nonlinear functions $f_{i}^{\eta_{i}}(z)$ (which would be present in $(\mathrm{Br})$ as well if only feedback transformations of the form $u=\beta(x) \tilde{u}$ had been considered), and the fact that, now, we have only $(m-1) \mathrm{z}$-chains (that will produce the $(m-1)$-chains of integrators for the static feedback lin- earizable reduced system), each z-chain being affected by the control vector field $\tilde{g}_{m}$ because of which the original system $\Sigma_{m}$ is not static feedback linearizable.

\section{Applications}

In this section, we present several examples in order to illustrate our main results. As explained in Section 3, there are systems that are at the same time linearizable via one-fold reduction and linearizable via invertible one-fold prolongation (Examples 1 and 2 below are such systems) but, in general, this is not the case (as shown by Example 3). We also highlight the fact the class of feedback transformations considered in this paper is smaller than the class of all noninvertible feedback transformations, see Section 2, where we explained that the difference between the two classes is the order in which we apply transformations. Example 4 presents a system that is not linearizable via a one-fold reduction but that becomes linearizable via a one-fold reduction that follows an initial modification of the drift.

Example 1. Consider the following control system

$$
\begin{aligned}
& \dot{x}_{1}=2 x_{3}+\sin x_{3}+x_{6} u_{4} \\
& \dot{x}_{2}=x_{5}+x_{3} u_{3}+x_{5} u_{4} \\
& \dot{x}_{3}=x_{6}+u_{4} \\
& \dot{x}_{4}=u_{3} \\
& \dot{x}_{5}=u_{2} \\
& \dot{x}_{6}=u_{1},
\end{aligned}
$$

around $x_{0}=0 \in \mathbb{R}^{6}$. We have $\mathcal{D}^{0}=\operatorname{span}\left\{\frac{\partial}{\partial x_{3}}+\right.$ $\left.x_{5} \frac{\partial}{\partial x_{2}}+x_{6} \frac{\partial}{\partial x_{1}}, \frac{\partial}{\partial x_{4}}+x_{3} \frac{\partial}{\partial x_{2}}, \frac{\partial}{\partial x_{5}}, \frac{\partial}{\partial x_{6}}\right\}$ and cork $\left(\mathcal{D}^{0} \subset\right.$ $\left.\mathcal{D}^{0}+\left[\mathcal{D}^{0}, \mathcal{D}^{0}\right]\right)=2$, thus if $\mathcal{D}^{0}$ contains an involutive subdistribution $\mathcal{N}^{0}$ of corank one, then $\mathcal{N}^{0}$ is unique. It is clear that $\mathcal{N}^{0}=\operatorname{span}\left\{\frac{\partial}{\partial x_{4}}+x_{3} \frac{\partial}{\partial x_{2}}, \frac{\partial}{\partial x_{5}}, \frac{\partial}{\partial x_{6}}\right\}$ and a straightforward calculation gives $\mathcal{N}^{1}=\operatorname{span}\left\{\frac{\partial}{\partial x_{2}}, \frac{\partial}{\partial x_{3}}, \frac{\partial}{\partial x_{4}}, \frac{\partial}{\partial x_{5}}, \frac{\partial}{\partial x_{6}}\right\}$ and $\mathcal{N}^{2}=T X$. So conditions (R1)-(R3) of Theorem 2 are satisfied and it follows that the above system is linearizable via one-fold reduction and, moreover, the to-beremoved control is $u_{4}$. Indeed, by introducing the following change of coordinates $z_{1}^{j}=L_{f}^{j-1} x_{1}$, for $1 \leq j \leq 3$, $z_{2}^{j}=L_{f}^{j-1}\left(x_{2}-x_{3} x_{4}\right)$, for $1 \leq j \leq 2$, and $z_{3}^{1}=x_{4}$, (where the functions $x_{1}$ and $x_{2}-x_{3} x_{4}$ are chosen such that in the new coordinates, the involutive distributions $\mathcal{N}^{i}$ are rectified), and applying a suitable invertible feedback transformation $u=\beta \tilde{u}$ (that does not change $u_{3}$ and $u_{4}$ ), we obtain

$$
\begin{array}{ll}
\dot{z}_{1}^{1}=z_{1}^{2}+a_{1}^{1}(z) \tilde{u}_{4} & \dot{z}_{2}^{1}=z_{2}^{2}+a_{2}^{1}(z) \tilde{u}_{4} \quad \dot{z}_{3}^{1}=\tilde{u}_{3}, \\
\dot{z}_{1}^{2}=z_{1}^{3}+a_{1}^{2}(z) \tilde{u}_{4} & \dot{z}_{2}^{2}=f_{2}^{2}(z)+\tilde{u}_{2}, \\
\dot{z}_{1}^{3}=f_{1}^{3}(z)+\tilde{u}_{1} &
\end{array}
$$

with $\tilde{u}_{4}=u_{4}$, and which, by putting $\tilde{u}_{4} \equiv 0$, clearly leads to a static feedback linearizable reduction. It can be shown that system (2) is also locally linearizable via an invertible onefold prolongation around $\left(x_{0}, u_{0}\right)=\left(0, u_{0}\right)$, where $u_{0} \in \mathbb{R}^{4}$ is such that $u_{40} \neq-1$ (see [15] where we discuss the issue of singular controls). For the sequence of involutive distributions $\mathcal{H}^{i}$ characterizing linearization via invertible one-fold prolongation, we necessarily have $\mathcal{H}^{0}=\mathcal{N}^{0}$ ( since $\mathcal{D}^{0}$ contains a unique involutive subdistribution of corank one) and, by a simple computation, $\mathcal{H}^{1}=T X$. Therefore, the to-beprolonged-control is $u_{4}$, as for the one-fold reduction. Notice that the only distributions of the sequences $\mathcal{N}^{i}$ and $\mathcal{H}^{i}$ that coincide are $\mathcal{N}^{0}$ and $\mathcal{H}^{0}$. 
Example 2. Consider the following control system

$\dot{x}_{1}^{1}=x_{1}^{2} \quad \dot{x}_{2}^{1}=x_{2}^{2} x_{4}^{1}+x_{2}^{2} u_{1} \dot{x}_{3}^{1}=x_{3}^{2} x_{4}^{1}+x_{3}^{2} u_{1} \dot{x}_{4}^{1}=u_{4}$, $\dot{x}_{1}^{2}=x_{4}^{1}+u_{1}, \dot{x}_{2}^{2}=u_{2}, \quad \dot{x}_{3}^{2}=u_{3}$

around $\left(x_{0}, u_{0}\right)$ where $x_{40}^{1} \neq 0$ and $x_{40}^{1}+u_{10} \neq 0$, for which we have $\mathcal{D}^{0}=\operatorname{span}\left\{\frac{\partial}{\partial x_{1}^{2}}+x_{2}^{2} \frac{\partial}{\partial x_{2}^{1}}+x_{3}^{2} \frac{\partial}{\partial x_{3}^{1}}, \frac{\partial}{\partial x_{2}^{2}}, \frac{\partial}{\partial x_{3}^{2}}, \frac{\partial}{\partial x_{4}^{1}}\right\}$, cork $\left(\mathcal{D}^{0} \subset \mathcal{D}^{0}+\left[\mathcal{D}^{0}, \mathcal{D}^{0}\right]\right)=2$ and $\mathcal{D}^{0}$ contains an (unique) involutive subdistribution of conrank one $\mathcal{N}^{0}=$ $\mathcal{H}^{0}=\operatorname{span}\left\{\frac{\partial}{\partial x_{2}^{2}}, \frac{\partial}{\partial x_{3}^{2}}, \frac{\partial}{\partial x_{4}^{1}}\right\}$. Notice that $a d_{f} g_{4}=g_{1}$ and, by a simple computation, we get $\mathcal{N}^{1}=\mathcal{H}^{1}=$ $\operatorname{span}\left\{\frac{\partial}{\partial x_{1}^{2}}, \frac{\partial}{\partial x_{2}^{1}}, \frac{\partial}{\partial x_{2}^{2}}, \frac{\partial}{\partial x_{3}^{1}}, \frac{\partial}{\partial x_{3}^{2}}, \frac{\partial}{\partial x_{4}^{1}}\right\}$ and $\mathcal{N}^{2}=\mathcal{H}^{2}=T X$. So all distributions $\mathcal{N}^{i}$ and $\mathcal{H}^{i}$ coincide, system (3) is both linearizable via a one-fold reduction and via an invertible one-fold prolongation and the to-be-removed control is the same as the to-be-prolonged-one: $u_{r}=u_{p}=u_{1}$.

Example 3. Consider the following control system, around $\left(x_{0}, u_{0}\right) \in \mathbb{R}^{8} \times \mathbb{R}^{3}$, with $u_{10} \neq-1$,

$$
\begin{array}{lll}
\dot{x}_{1}^{1}=x_{1}^{2}+\left(x_{1}^{3}\right)^{2}+x_{2}^{1} & \dot{x}_{2}^{1}=x_{2}^{2} & \dot{x}_{3}^{1}=x_{3}^{2}+x_{3}^{2} u_{1} \\
\dot{x}_{1}^{2}=x_{1}^{3}+x_{1}^{1} & \dot{x}_{2}^{2}=x_{2}^{3}+x_{2}^{3} u_{1} & \dot{x}_{3}^{2}=u_{3}, \\
\dot{x}_{1}^{3}=x_{1}^{2}+u_{1}, & \dot{x}_{2}^{3}=u_{2}, &
\end{array}
$$

for which $\mathcal{D}^{0}=\operatorname{span}\left\{\frac{\partial}{\partial x_{1}^{3}}+x_{2}^{3} \frac{\partial}{\partial x_{2}^{2}}+x_{3}^{2} \frac{\partial}{\partial x_{3}^{1}}, \frac{\partial}{\partial x_{2}^{3}}, \frac{\partial}{\partial x_{3}^{2}}\right\}$, $\operatorname{cork}\left(\mathcal{D}^{0} \subset \mathcal{D}^{0}+\left[\mathcal{D}^{0}, \mathcal{D}^{0}\right]\right)=r=2$ and $\mathcal{D}^{0}$ contains an (unique) involutive subdistribution of conrank one $\mathcal{N}^{0}=\operatorname{span}\left\{\frac{\partial}{\partial x_{2}^{3}}, \frac{\partial}{\partial x_{3}^{2}}\right\}$. It is easy to compute the sequence of distributions $\mathcal{N}^{i}$, all of them are involutive and $\mathcal{N}^{5}=$ $T X$. Hence, according to Theorem 2, system (4) is locally linearizable via one-fold reduction and the to-be-removedcontrol is $u_{1}$. The new coordinates in which the reduced system (obtained by putting $u_{1} \equiv 0$ ) is in the Brunovský canonical form (with two-chains of integrators only) are $z_{1}^{j}=L_{f}^{j-1} x_{1}^{3}$, for $1 \leq j \leq 6$, and $z_{2}^{j}=x_{3}^{j}$, for $1 \leq j \leq 2$. Contrary to the previous examples, system (4) is not linearizable via invertible one-fold prolongation. In order to see it, compute the sequence of distributions $\mathcal{H}^{i}$. We have $\mathcal{H}^{0}=\mathcal{N}^{0}=\operatorname{span}\left\{\frac{\partial}{\partial x_{2}^{3}}, \frac{\partial}{\partial x_{3}^{2}}\right\}$ (because the involutive subdistribution of conrank one of $\mathcal{D}^{0}$ is unique). The distribution $\mathcal{H}^{1}=\operatorname{span}\left\{\frac{\partial}{\partial x_{1}^{3}}, \frac{\partial}{\partial x_{2}^{2}}, \frac{\partial}{\partial x_{2}^{3}}, \frac{\partial}{\partial x_{3}^{1}}, \frac{\partial}{\partial x_{3}^{2}}\right\}$ is involutive, but $\mathcal{H}^{2}=\mathcal{H}^{1}+\operatorname{span}\left\{\frac{\partial}{\partial x_{1}^{2}}+2 x_{1}^{3} \frac{\partial}{\partial x_{1}^{1}}, \frac{\partial}{\partial x_{2}^{1}}\right\}$ is clearly not. Thus system (4) cannot be linearizable via invertible one-fold prolongation although it is so via a one-fold reduction.

Example 4. Consider around $x_{0}=0 \in \mathbb{R}^{7}$ :

$$
\begin{array}{lll}
\dot{x}_{1}^{1}=x_{2}^{1} & \dot{x}_{2}^{1}=x_{2}^{2} & \dot{x}_{3}^{1}=x_{3}^{2}+x_{3}^{2} u_{1} \\
\dot{x}_{1}^{2}=x_{1}^{1}+x_{2}^{3} x_{3}^{2}+u_{1}, & \dot{x}_{2}^{2}=x_{2}^{3}+x_{2}^{3} u_{1} & \dot{x}_{3}^{2}=u_{3}, \\
& \dot{x}_{2}^{3}=u_{2}, &
\end{array}
$$

for which $\mathcal{D}^{0}=\operatorname{span}\left\{\frac{\partial}{\partial x_{1}^{2}}+x_{2}^{3} \frac{\partial}{\partial x_{2}^{2}}+x_{3}^{2} \frac{\partial}{\partial x_{3}^{1}}, \frac{\partial}{\partial x_{2}^{3}}, \frac{\partial}{\partial x_{3}^{2}}\right\}$, cork $\left(\mathcal{D}^{0} \subset \mathcal{D}^{0}+\left[\mathcal{D}^{0}, \mathcal{D}^{0}\right]\right)=2$ and $\mathcal{D}^{0}$ contains an (unique) involutive subdistribution of conrank one $\mathcal{N}^{0}=$ $\operatorname{span}\left\{\frac{\partial}{\partial x_{2}^{3}}, \frac{\partial}{\partial x_{3}^{2}}\right\}$. The distribution $\mathcal{N}^{1}=\operatorname{span}\left\{\frac{\partial}{\partial x_{2}^{2}}+\right.$ $\left.x_{3}^{2} \frac{\partial}{\partial x_{1}^{2}}, \frac{\partial}{\partial x_{3}^{1}}+x_{2}^{3} \frac{\partial}{\partial x_{1}^{2}}, \frac{\partial}{\partial x_{2}^{3}}, \frac{\partial}{\partial x_{3}^{2}},\right\}$ is not involutive, and according to Theorem 2, the above system is not feedback linearizable via one-fold reduction. Now recall that when we defined the linearization via one-fold reduction, we did not consider the most general class of feedback transformations: we only allow to change the control vector fields of the original system (the original drift being preserved). Observe that, by changing also the drift, system (5) becomes linearizable via one-fold reduction. Indeed, by applying the invertible feedback transformation $u_{1}=-x_{2}^{3} x_{3}^{2}+\tilde{u}_{1}, u_{2}=\tilde{u}_{2}$ and $u_{3}=\tilde{u}_{3}$ (that changes the drift as $f \mapsto f-x_{2}^{3} x_{3}^{2} g_{1}$ ), we get $\begin{aligned} \dot{x}_{2}^{1} & =x_{2}^{2} \\ \dot{x}_{1}^{1}=x_{2}^{1} & x_{1}^{1}+\tilde{u}_{1}, \dot{x}_{2}^{2}=x_{2}^{3}-\left(x_{2}^{3}\right)^{2} x_{3}^{2}+x_{2}^{3} \tilde{u}_{1} \dot{x}_{3}^{1}=x_{3}^{2}-x_{2}^{3}\left(x_{3}^{2}\right)^{2}+x_{3}^{2} \tilde{u}_{1} \\ \dot{x}_{2}^{3}=\tilde{u}_{2}, & \dot{x}_{3}^{2}=\tilde{u}_{3},\end{aligned}$

for which all distributions $\mathcal{N}^{i}$ are involutive and $\mathcal{N}^{4}=T X$, and, thus system (6) is linearizable via one-fold reduction.

\section{References}

[1] R.W. Brockett. Feedback invariants for nonlinear systems. IFAC Congress 6, Helsinki, pages 1115-1120, 1979.

[2] R. Bryant. Some aspects of the local and global theory of Pfaffian systems. PhD thesis, University of North Carolina at Chapel Hill, 1979.

[3] B. Charlet, J. Lévine, and R. Marino. Sufficient conditions for dynamic state feedback linearization. SIAM J. Control Optim., 29(1):38-57, 1991.

[4] D. Cheng. Linearization with dynamic compensation. J. Syst. Sci. Math. Sci., 7(3):200-2004, 1987.

[5] M. Fliess, J. Lévine, P. Martin, and P. Rouchon. Flatness and defect of non-linear systems: introductory theory and examples. Internat. J. Control, 61(6):1327-1361, 1995.

[6] M. Fliess, J. Lévine, P. Martin, and P. Rouchon. A LieBäcklund approach equivalence and flatness of nonlinear systems. IEEE Trans. Automat. Control, 44(5):922-937, 1999.

[7] S.S. Ge, Z. Sun, and T.H. Lee. Nonregular feedback linearization for a class of second-order nonlinear systems. Automatica, 37(11):1819 - 1824, 2001.

[8] L. Hunt and R. Su. Linear equivalents of nonlinear time varying systems. In Proc. MTNS, Santa Monica, CA, pages 119$123,1981$.

[9] A. Isidori, C.H. Moog, and A. De Luca. A sufficient condition for full linearization via dynamic state feedback. In Proc. CDC, volume 25, pages 203-208. IEEE, 1986.

[10] B. Jakubczyk. Invariants of dynamic feedback and free systems. In Proc. ECC, pages 1510-1513, 1993.

[11] B. Jakubczyk and W. Respondek. On linearization of control systems. Bull. Acad. Polonaise Sci. Ser. Sci. Math., pages 517-522, 1980

[12] P. Martin, P. Rouchon, and R. Murray. Flat systems, equivalence and trajectory generation, CDS Technical Report, Caltech. 2003.

[13] F. Nicolau, S. Li, and W. Respondek. Linearization via onefold reduction of multi-input control systems. In preparation.

[14] F. Nicolau and W. Respondek. Two-inputs control-affine systems linearizable via one-fold prolongation and their flatness. Eur. J. Control, 28:20-37, 2016.

[15] F. Nicolau and W. Respondek. Flatness of multi-input controlaffine systems linearizable via one-fold prolongation. SIAM J. Control and Optim., 55(5):3171-3203, 2017.

[16] W. Pasillas-Lépine and W. Respondek. Contact systems and corank one involutive subdistributions. Acta Applicandae Mathematica, 69(2):105-128, 2001.

[17] J.B. Pomet. A differential geometric setting for dynamic equivalence and dynamic linearization. Banach Center Publ., Vol. 32, pages 319-339, 1995.

[18] W. Respondek. Symmetries and minimal flat outputs of nonlinear control systems. In New Trends in Nonlinear Dynamics and Control and their Applications, volume LNCIS 295, pages 65-86. Springer, 2003.

[19] Z. Sun and X. Xia. On nonregular feedback linearization. Automatica, 33(7):1339 - 1344, 1997.

[20] J. Zhong, M. Karasalo, D. Cheng, and X. Hu. New results on non-regular linearization of non-linear systems. Internat. $J$. Control, 80(10):1651-1664, 2007. 